\title{
MDM2 SNP309 and p53 Arg72Pro in cutaneous melanoma: association between SNP309 GG genotype and tumor Breslow thickness
}

\begin{abstract}
Mario Capasso $^{1}$, Fabrizio Ayala ${ }^{2}$, Rosa Anna Avvisati ${ }^{1}$, Roberta Russo ${ }^{1}$, Antonella Gambale ${ }^{1}$, Nicola Mozzillo ${ }^{2}$, Paolo Antonio Ascierto ${ }^{2}$ and Achille Iolascon ${ }^{1,3}$

A functional single nucleotide polymorphism (SNP) $309 \mathrm{~T} / \mathrm{G}$ within mouse double minute 2 (MDM2) gene has been linked to onset and outcome of disease in tumors. Two published studies have shown discordant results regarding the effect of this SNP on age at diagnosis of cutaneous melanoma (CM) in Caucasian female populations. Here, we examined the age at diagnosis and clinical associations of CM with SNP309 and the related polymorphism, p53 Arg72Pro, in an Italian population (249 CM patients and 291 cancer-free controls) composed of women and men. MDM2 intronic region of $294 \mathrm{bp}$ was directly sequenced, whereas Arg72Pro SNP was analyzed by polymerase chain reaction-restriction fragment length polymorphism. No associations were found among the SNP309, Arg72Pro, risk of CM, age at diagnosis and presence of metastasis in total subjects and when stratified according to the gender. The SNP309 was significantly associated with tumor Breslow thickness. The $P$-value in the minor allele recessive mode was 0.02 , and the odds ratio (OR) adjusted for gender and age was 3.11 (95\% confidential interval $(\mathrm{Cl})=1.21-8.00)$. The SNP309 is not associated with the risk and age of onset of CM, and the presence of metastasis in an Italian population but the SNP309 GG may be a risk genotype for increasing in tumor Breslow thickness. Journal of Human Genetics (2010) 55, 518-524; doi:10.1038/jhg.2010.62; published online 10 June 2010
\end{abstract}

Keywords: Breslow thickness; cutaneous melanoma; MDM2; p53; SNP

\section{INTRODUCTION}

The human homolog of mouse double minute 2 (MDM2) is a negative regulator of the tumor protein p53 (TP53). MDM2 is a nuclear phosphoprotein that binds to TP53 and inhibits TP53-dependent transcription. ${ }^{1}$ Overexpression of this gene can result in inactivation of TP53, diminishing its tumor suppressor function. ${ }^{2}$ MDM2 has also been shown to promote tumor growth in a TP53-independent manner. ${ }^{3}$ A single nucleotide polymorphism (SNP) within intron 1 of MDM2, a T-to-G substitution (T/G) at position 309 (SNP309) (rs2279744), has been shown to lead to enhanced binding of the Sp1 transcription factor, resulting in elevated levels of both MDM2 mRNA and protein, thereby attenuating the p53 response. ${ }^{4}$ In Li-Fraumeni patients, individuals homozygous or heterozygous for SNP309 (GG or TG) were shown to develop cancer at an earlier age than wild-type individuals; in patients with sporadic soft tissue sarcomas, the 309 GG genotype correlated with an average 12-year earlier age of diagnosis. ${ }^{4}$ SNP309 was thus postulated as a potential modulator of cancer susceptibility. ${ }^{4}$ Since the publication of the original report, ${ }^{4}$ a number of studies have explored whether SNP309 was associated with the risk or the age of onset of different types of cancer. These studies of the
SNP309 polymorphism have demonstrated variable frequencies of SNP309 GG depending on race and ethnicity. ${ }^{5-8}$ Similarly, the association between SNP309 and the development of cancer has produced conflicting data. 9

A recent study in cutaneous melanoma (CM) found that the MDM2 SNP309 GG genotype correlated with earlier onset age. ${ }^{10}$ An other study reported no significant association with age of diagnosis and risk of CM. ${ }^{11}$ Both studies were performed only on Caucasian women. Several studies in other tumors have examined the association between MDM2 SNP309 and a well-studied polymorphism in p53, Arg72Pro (rs1042522; R/P). The results have shown positive associations between the proline/proline (PP) genotype and disease risk for some tumor types (for example esophageal squamous cell carcinoma, lung cancer, renal cell carcinoma ${ }^{12-14}$ ) but no association for other tumor types (for example colorectal cancer, breast cancer ${ }^{15,16}$ ). The association between the p53 Arg72Pro polymorphism itself and melanoma risk is controversial, ${ }^{17-20}$ with some studies showing associations between melanoma risk and the PP genotype, ${ }^{17,19}$ but others showing increased melanoma risk with the arginine/arginine (RR) genotype. ${ }^{18,20}$

${ }^{1}$ CEINGE Biotecnologie Avanzate, Napoli, Italy; ${ }^{2}$ Istituto Nazionale dei Tumori Fondazione G. Pascale, Via Mariano Semmola, Napoli, Italy and ${ }^{3}$ Dipartimento di Biochimica e Biotecnologie Mediche, Università degli Studi di Napoli 'Federico II', Napoli, Italy

Correspondence: Professor A Iolascon, Dipartimento di Biochimica e Biotecnologie Mediche, University of Naples Federico II, CEINGE Biotecnologie Avanzate s.c.ar.l., Via Comunale Margherita 482, Napoli 80145, Italy.

E-mail: iolascon@ceinge.unina.it

Received 11 January 2010; revised 8 April 2010; accepted 2 May 2010; published online 10 June 2010 
In light of these findings, we examined the MDM2 SNP309 and p53 Arg72Pro genotypes to determine whether they could be linked to an increased CM susceptibility, age of cancer diagnosis and clinical pathological variables in an Italian population composed of women and men.

\section{MATERIALS AND METHODS}

\section{Subjects}

For this case-control study, 249 patients with melanoma (102 males and 147 females; median age 37.0 years (13.0)) were tested. Patient data were compared with data from 291 cancer-free controls (110 males and 181 females; median age 37.0 years (12.0)). Patients and healthy controls enrolled in the study originated from Campania, a region of Southern Italy. The patients were diagnosed with CM at the Department of Dermatology, National Institute of Tumors G. Pascale between 2000 and 2007, of whom blood for DNA preparation was available, were included in our study. Clinical data (including sex, age, Breslow thickness and presence of metastasis) were retrieved from patients' medical records. Our sample consisted of 73 melanoma in situ and 176 invasive CM with median tumor Breslow thickness of $1.00 \mathrm{~mm}$ (1.50). Thirty-three patients presented with metastases (26 with only lymph node metastasis and 7 with lymph node and visceral metastasis). The primary tumor and any signs of progression were determinate according to recent classifications detected and confirmed by sonography, computed tomography or histopathology after surgical removal. ${ }^{21}$ Given that people from Campania are exposed to the sun frequently, $40 \%$ of children have sunburns during holidays and $71.2 \%$ stay in the sun from 1100 to 1600 hours, ${ }^{22}$ we included in this study only the cases who had more than three sunburns defined as episodes of intense erythema with or without blisters causing pain and discomfort for $>2$ days. ${ }^{23}$ This explains the low-median age (37.0 years) of our patients. The cancer-free control subjects were recruited during the same period from cancerfree visitors to the Department of Dermatology, National Institute of Tumors G. Pascale who were not seeking medical care but instead accompanied patients to our outpatient clinics and were frequency matched to patients with CM by age, sex, history of sunburns and ethnicity and not blood related to the patients. They were free of clinically manifest disease in their personal history, and free of malignant skin diseases at the time of inspection served as healthy populationbased controls. This study was approved by the local ethics committee.

\section{Genotyping}

Genomic DNA was prepared from blood using Wizard Genomic DNA purification kit (Promega, Milan, Italy). The genotyping of MDM2 SNP309 (rs2279744) was performed by direct sequencing. Briefly, a fragment of the MDM2 intronic region of $294 \mathrm{bp}$ was amplified from genomic DNA by polymerase chain reaction (PCR) in a $25-\mu \mathrm{l}$ volume with Master Mix (Promega). The oligonucleotide primers were designed from the intronic sequence of the human MDM2 (forward 5'-GTTTTGTTGGACTGGGGCTA- ${ }^{\prime}$, reverse 5'-TCGGAACGTGTC TGAACTTG-3'). The PCR products were checked by DNA agarose gel electrophoresis. Direct sequencing was performed using the Dye Terminator Cycle Sequencing FS Ready Reaction Kit (Perkin-Elmer Applied Biosystems, Waltham, MA, USA) and a 373A DNA sequencer (Applied Biosystems, Branchberg, NJ, USA). On sequencing MDM2 intronic amplicon (294 bp), an additional already reported SNP (344 A/T; rs119633) was detected in the control and $\mathrm{CM}$ populations. Genotypes of p53 Arg72Pro ( $r 1042522$; R/P) were determined by restriction fragment length polymorphism analysis using BstUI (New England Biolabs, Ipswich, MA, USA). A fragment of the p53-coding region of $313 \mathrm{bp}$ was amplified from genomic DNA by PCR as above described using the primers: forward 5'-GTCCCAAGCAATGGATGATT-3', reverse $5^{\prime}$-CAGGCATTGAAGTCTCATGG-3'. The restriction fragment length polymorphism assay was performed in $12 \mu \mathrm{l}$ reaction volume containing the PCR product and the restriction enzyme. In the presence of the p53 $\mathrm{P}$ allele, the enzyme cut its 313-bp PCR product into two bands of 205 and $108 \mathrm{bp}$.

\section{Statistical methods}

The characteristics of CM patients were summarized using medians with interquartile range for continuous variables and percentages for categorical variables. Comparison of genotypic frequencies between groups was performed by $\chi^{2}$ test. Odds ratios (ORs) and $95 \%$ confidence intervals (CIs) were calculated to assess the relative disease risk conferred by a specific genotype. Genotype frequencies were tested for agreement with Hardy-Weinberg equilibrium using $\chi^{2}$ test based on the likelihood theory, using estimates of allele frequencies. A logistic regression was used to determine whether the SNP309 genotype was an independent predictor of the increase in tumor thickness. The potential confounding factors age and gender were evaluated. Associations between genotype and age of cancer diagnosis, and Breslow thickness ( $\mathrm{mm}$ ) were determined using Mann-Whitney $U$ test. No adjustments were made for multiple comparisons. A two-sided $P$-value $<0.05$ was considered statistically significant.

\section{RESULTS}

SNP309 and Arg72Pro frequencies in a control and CM population We investigated the MDM2 SNP309 and p53 Arg72Pro polymorphisms in 249 CM cases and 291 healthy controls of Italian origin. As a result of frequency matching, no statistically significant differences in the frequency distribution of age or sex between patients and control subjects were found. Five samples of the cases group for the MDM2 SNP 309, seven samples of the controls and nine of the cases group for p53 Arg72Pro failed genotyping because of low DNA quality. The alleles were in Hardy-Weinberg equilibrium in the controls $(P>0.05)$. The allele frequencies for both SNPs (G SNP309 41\% and P Arg72Pro $30 \%$ ) in the control group were similar with others previously reported in larger Caucasian study populations. ${ }^{9,18}$

We found no association among SNP309, p53 Arg72Pro and risk of CM (Table 1; $P=0.95$ and $P=0.13$ ). As the SNP309 has been also shown to influence the cancer onset in gender-specific manner, ${ }^{4}$ we stratified our population according to the sex. No association was found in both subgroups (women and men) for both SNPs (Table 1). The genotype frequencies of SNP309 in women subgroup between controls and cases were very similar $(P=0.83)$. These results are in accordance with those reported in the study on a Caucasian women population composed of 851 healthy controls (TT 380 (45.0\%), TG $356(42.0 \%)$ and GG $115(14.0 \%)$ ) and $218 \mathrm{CM}$ patients (TT 97 (45.0\%), TG $92(42.0 \%)$ and GG $29(13.0 \%))$, where no risk for CM $(P=0.99)$ was found and the genotype frequencies of controls and cases were very similar. ${ }^{11}$

In the analysis on the combination of the two polymorphisms, individuals with MDM2 SNP309 TT and TG genotypes, and p53 RR and RP genotypes were combined. No significant association was found in all of three groups: total, women and men (Table 2).

The frequencies of the genotypes TT, TA, AA of the SNP $344 \mathrm{~A} / \mathrm{T}$ detected by the sequencing analysis were $280(96.2 \%), 11(3.8 \%), 0$ $(0.0 \%)$, and $234(95.9 \%), 10(4.1 \%), 0(0.0 \%)$ in controls and cases, respectively. As the minor allele frequency was low (A allele $1.9 \%$ in controls), this SNP was excluded from further analyses. However, the genotype frequencies were similar to those observed in an other Caucasian population; TT $266(96.7 \%)$, TA $9(3.3 \%)$, AA $0(0.0 \%){ }^{24}$

\section{SNP309, Arg72Pro and age of CM diagnosis}

The two above-mentioned studies ${ }^{10,11}$ have reported controversial results about the association between SNP309 and age at diagnosis of $\mathrm{CM}$ in female populations of Caucasian origin. So, to test whether SNP309 and Arg72Pro were associated with an earlier age of cancer onset in an Italian population, the age at first diagnosis of CM was recorded and median values plotted with respect to genotype in total patients and stratified by gender (Figure 1). No statistically significant differences were observed for both SNPs. The median age (years) at diagnosis according to TT, TG, GG genotypes of MDM2 was 38.0 (13.0), 37.0 (13.0), 38.0 (14.0) for total patients, 37.0 (15.0), 
Table 1 Genotype frequencies of MDM2 SNP309 and p53 Arg72Pro in controls and patients with $\mathrm{CM}$

\begin{tabular}{|c|c|c|c|}
\hline Genotype & Controls n (\%) & Cases n (\%) & P-value \\
\hline \multicolumn{4}{|l|}{ Total } \\
\hline \multicolumn{4}{|c|}{ MDM2 SNP309 } \\
\hline TT & $107(36.8)$ & $87(35.7)$ & \\
\hline $\mathrm{TG}$ & $128(44.0)$ & $108(44.3)$ & \\
\hline GG & $56(19.2)$ & $49(20.1)$ & 0.95 \\
\hline \multicolumn{4}{|c|}{ p53 Arg72Pro } \\
\hline $\mathrm{RR}$ & 139 (48.9) & $123(51.3)$ & \\
\hline $\mathrm{RP}$ & $122(43.0)$ & $87(36.3)$ & \\
\hline PP & $23(8.1)$ & $30(12.5)$ & 0.13 \\
\hline \multicolumn{4}{|l|}{ Women } \\
\hline \multicolumn{4}{|c|}{ MDM2 SNP309 } \\
\hline $\mathrm{TT}$ & $58(32.0)$ & $51(34.9)$ & \\
\hline $\mathrm{TG}$ & $85(47.0)$ & $64(43.8)$ & \\
\hline $\mathrm{GG}$ & $38(21.0)$ & $31(21.2)$ & 0.83 \\
\hline \multicolumn{4}{|c|}{ p53 Arg72Pro } \\
\hline $\mathrm{RR}$ & 87 (48.9) & $75(52.4)$ & \\
\hline $\mathrm{RP}$ & $73(41.0)$ & 47 (32.9) & \\
\hline PP & $18(10.1)$ & $21(14.7)$ & 0.23 \\
\hline \multicolumn{4}{|l|}{ Men } \\
\hline \multicolumn{4}{|c|}{ MDM2 SNP309 } \\
\hline $\mathrm{TT}$ & $49(44.5)$ & $36(36.7)$ & \\
\hline $\mathrm{TG}$ & $43(39.1)$ & $44(44.9)$ & \\
\hline $\mathrm{GG}$ & $18(16.4)$ & $18(18.4)$ & 0.52 \\
\hline \multicolumn{4}{|c|}{ p53 Arg72Pro } \\
\hline $\mathrm{RR}$ & $52(49.1)$ & $48(49.5)$ & \\
\hline $\mathrm{RP}$ & $49(46.2)$ & $40(41.2)$ & \\
\hline PP & $5(4.7)$ & $9(9.3)$ & 0.40 \\
\hline
\end{tabular}

Abbreviations: CM, cutaneous melanoma; MDM2, mouse double minute 2; SNP, single nucleotide polymorphism.

$P$-value: $\chi^{2}$ on genotype frequencies.

Table 2 Risk of CM according to genotypes of MDM2 SNP309 and p53 Arg72Pro polymorphisms

\begin{tabular}{lcccc}
\hline MDM2 SNP309 & $p 53$ Arg72Pro & Controls/cases & $P$ & OR $(95 \%$ Cl) \\
\hline Total & & & & \\
TT/TG & RR/RP & $208 / 170$ & & 1 \\
GG & RR/RP & $53 / 39$ & 0.65 & $0.90(0.57-1.43)$ \\
TT/TG & PP & $20 / 24$ & 0.23 & $1.47(0.78-2.75)$ \\
GG & PP & $3 / 6$ & 0.19 & $2.45(0.60-9.93)$ \\
& & & & \\
Women & & & & \\
TT/TG & RR/RP & $124 / 98$ & & \\
GG & RR/RP & $36 / 23$ & 0.48 & $0.81(0.45-1.45)$ \\
TT/TG & PP & $16 / 16$ & 0.53 & $1.26(0.60-2.66)$ \\
GG & PP & $2 / 5$ & 0.15 & $3.16(0.60-16.6)$ \\
& & & & \\
Men & & & & \\
TT/TG & RR/RP & $84 / 72$ & & 1 \\
GG & RR/RP & $17 / 16$ & 0.81 & $1.10(0.52-2.33)$ \\
TT/TG & PP & $4 / 8$ & 0.17 & $2.33(0.67-8.07)$ \\
GG & PP & $1 / 1$ & 0.91 & $1.17(0.07-18.98)$ \\
\hline
\end{tabular}

Abbreviations: $\mathrm{Cl}$, confidence interval; $\mathrm{CM}$, cutaneous melanoma; MDM2, mouse double minute 2; OR, odds ratio; SNP, single nucleotide polymorphism.

$P$-value: $\chi^{2}$ test on genotype frequencies.
35.0 (20.0), 40.0 (16.0) for women patients and 38.5 (9.0), 37.0 (10.0) and 37.0 (15.0) for men patients, respectively. Although according to RR, RP, PP genotypes of p53 Arg72Pro, it was 38.0 (13.0), 37.0 (11.0), 36.0 (17.0) for total patients, 38.0 (16.0), 35.0 (14.0), 33.0 (20.0) for women patients and 38.0 (10.0), 37.0 (12.0) and 37.0 (13.0) for men patients, respectively.

\section{SNP309 and Arg72Pro and clinic pathological characteristics}

We examined SNP309 and Arg72Pro genotypes with respect to Breslow thickness and the presence of metastasis in 176 patients with invasive CM. The results are shown in Table 3. When stratifying patients according to clinically significant Breslow thickness of $0.75 \mathrm{~mm},{ }^{25}$ the SNP309 GG genotype was significantly associated with tumors thicker than $0.75 \mathrm{~mm}$ with an OR of $3.18(95 \% \mathrm{CI}=1.18-$ 8.55, $P=0.02$ ) compared with TG and of 3.11 (95\% $\mathrm{CI}=1.12-8.64$, $P=0.02)$ compared with TT genotype. When TG was compared with TT genotype, we found no significant association $(\mathrm{OR}=1.00,95 \%$ $\mathrm{CI}=0.50-2.00, P=1.00)$. Assuming a recessive model (GG vs TG+TT), we observed a significant increase of GG frequencies among patients with a Breslow thickness $>0.75 \mathrm{~mm}(\mathrm{OR}=3.11,95 \% \mathrm{CI}=1.21-8.00$, $P=0.01)$. Taken together, these results support a recessive penetrance for the $\mathrm{G}$ allele. A logistic regression analysis was used to show that the association, in the recessive model, between SNP309 GG genotype and the increase in tumor thickness was independent factor of other confounding variables such as age and gender. Correction for these variables changed the $P$-value from 0.01 (without correction) to $0.02(\mathrm{OR}=3.17,95 \% \mathrm{CI}=1.22-8.21)$ after correction. Accordingly, subgroup analysis revealed that the SNP309 genotypes in female and male patients had the same association trend with Breslow thickness (Table 3). The difference in $P$-values is probably due to the difference of the number of subjects between the two subgroups. The Arg72Pro genotype frequencies showed no significant association with Breslow thickness (Table 3 ).

In addition, median tumor Breslow thickness was compared among individuals of different genotypes for SNP309. The median tumor thickness of TT, TG and GG genotype were $0.90 \mathrm{~mm}$ (1.00), $0.98 \mathrm{~mm}$ (1.89) and $1.45 \mathrm{~mm}$ (1.60). The increased median tumor thickness in GG compared with TT+TG genotype was statistically significant $(P=0.03)$. Moreover, Firoz et al. found that the Breslow thickness median was higher in GG subjects than TT subjects for both women and men, but this difference was not statistically significant. ${ }^{10}$

No significant associations with metastasis were found in total patients (Table 3). There was a significant decreased risk to have metastasis for women with TG or RP genotype, but given the low number of patients with metastasis this result should be taken with caution.

\section{DISCUSSION}

The MDM2 SNP309 and p53 Arg72Pro polymorphisms were examined in an Italian population of $249 \mathrm{CM}$ patients and 291 cancer-free control to establish any associations between SNP309, Arg72Pro genotypes and CM. In CM cohort both SNPs were also analyzed with respect to age at cancer diagnosis and pathological variables. Our results showed no association between SNP309, Arg72Pro genotypes and the risk of CM. On the other hand the SNP309 GG genotype was associated with thick tumors, but no effect was found on age at cancer diagnosis and metastasis. Genotypes of SNP309, the closest SNP344 and Arg72Pro were distributed in accordance with Hardy-Weinberg equilibrium in controls and genotype frequencies were in broad agreement with those in previously published studies of Caucasian subjects. ${ }^{9,18,24}$ This provides additional validation of both the 

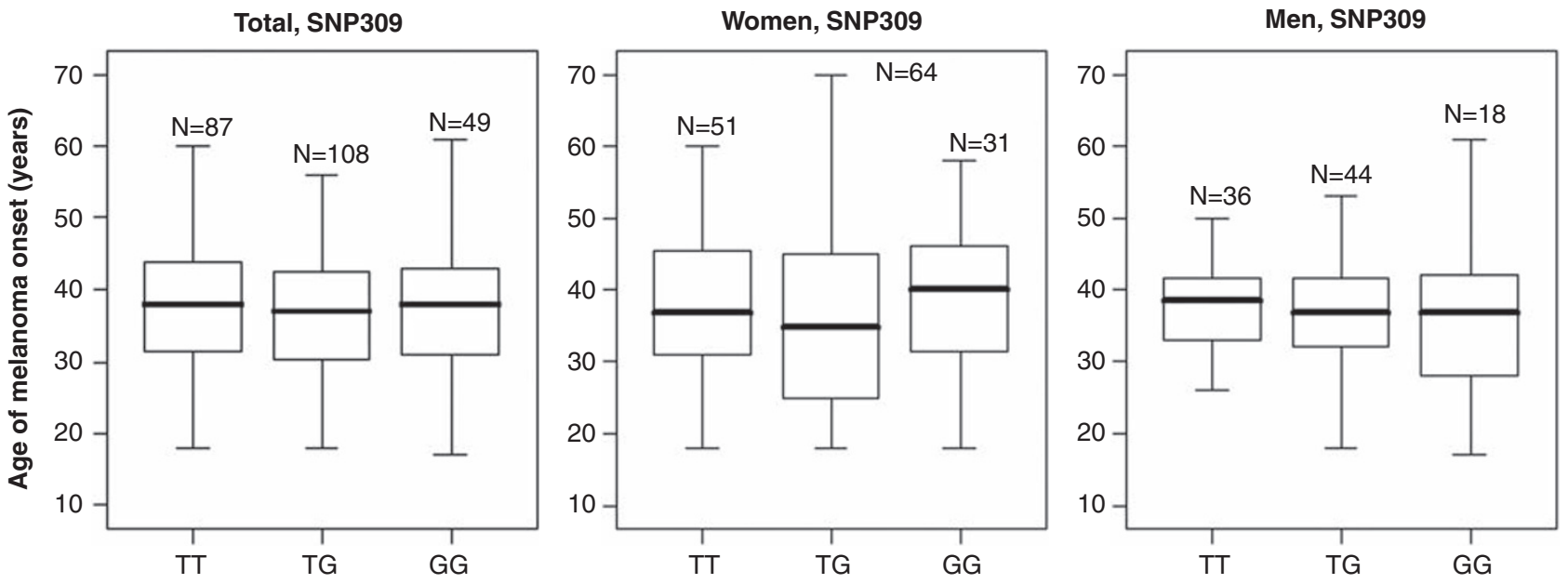

Total, Arg72Pro
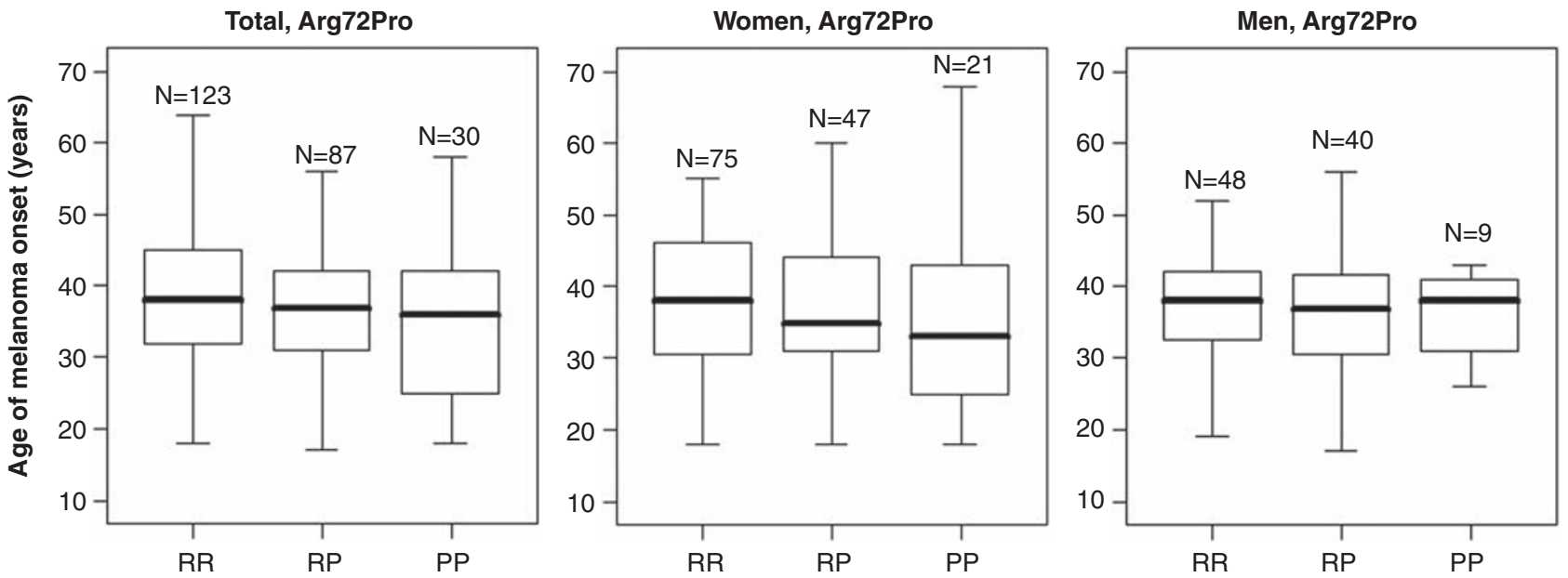

Figure 1 Box whiskers plot showing the age distribution of cutaneous melanoma diagnosis in total melanoma population and categorized by gender with respect to MDM2 SNP309 and p53 Arg72Pro genotype. Median values are represented by a solid horizontal bar, boxes denote the interquartile range and bars indicate the maximum and minimum values. Shown above is the number of subjects per group.

genotyping methodology and representative nature of the control group used in this study.

Melanoma risk is determined by a large number of variables. We have recently demonstrated that a predict functional SNP of BMP4 could influence the susceptibility to $\mathrm{CM}^{26}$ and other genetic associations with the risk of $\mathrm{CM}$ have been found. ${ }^{27}$ The association between MDM2 SNP309 and tumor risk has been evaluated in diverse populations including diverse tumor types (lung, breast, colorectal, bladder, ovarian and so on). ${ }^{9}$ To our knowledge, this is the first study investigating the role of MDM2 SNP309 polymorphism in CM patients and healthy controls composed of women and men. Recently, an analysis of this polymorphism and skin cancer risk was published using patients and controls from the Nurses Health Study. ${ }^{11}$ Using a women population of 218 melanoma patients and 851 controls, no significant associations were observed between SNP309, Arg72Pro and risk of CM. ${ }^{11}$ There were also no statistically significant associations with risk of basal cell carcinoma or squamous cell carcinoma. ${ }^{11}$ These results are in accordance with ours. Of note, the SNP309 genotype frequencies between women cases and women controls in our and their population were very similar. This strengths the evidence of non-association between SNP309 and CM development. In addition, we also observed no genetic association among men and when all subjects (women and men) were pooled. Taken together, our results and literature data support the evidence that the MDM2 SNP309 polymorphism does not influence the risk of $\mathrm{CM}$ in women and in men of Caucasian origin.

In an other study, the MDM2 SNP309 was associated with an earlier age of $\mathrm{CM}$ in women. ${ }^{10}$ The greatest $\mathrm{OR}$ for the diagnosis of $\mathrm{CM}$ among women with a SNP309 GG genotype was for those $<40$ years of age. ${ }^{10}$ Here, we observed no association with age at diagnosis both among women and men even when an estimate of the risk of $\mathrm{CM}$ by age group was performed (data not shown). Also the abovementioned study ${ }^{11}$ found no significant associations among the MDM2 SNP309 and age of diagnosis in women with CM as well as in women with basal cell carcinoma or squamous cell carcinoma. Statistically robust observations made in six independent patient populations and four different tumor types (diffuse large B-cell lymphoma, soft tissue sarcoma, invasive ductal carcinoma of the breast and colorectal cancer) support the hypothesis that MDM2 SNP309 GG genotype may accelerate tumor formation in a gender-specific and hormonaldependent manner. ${ }^{28,29}$ In these studies, the differences in tumor onset were observed when patients were divided into 'premenopausal' and 'postmenopausal' groups. The role of this polymorphism in influencing the age at diagnosis of CM remains controversial. It should be 


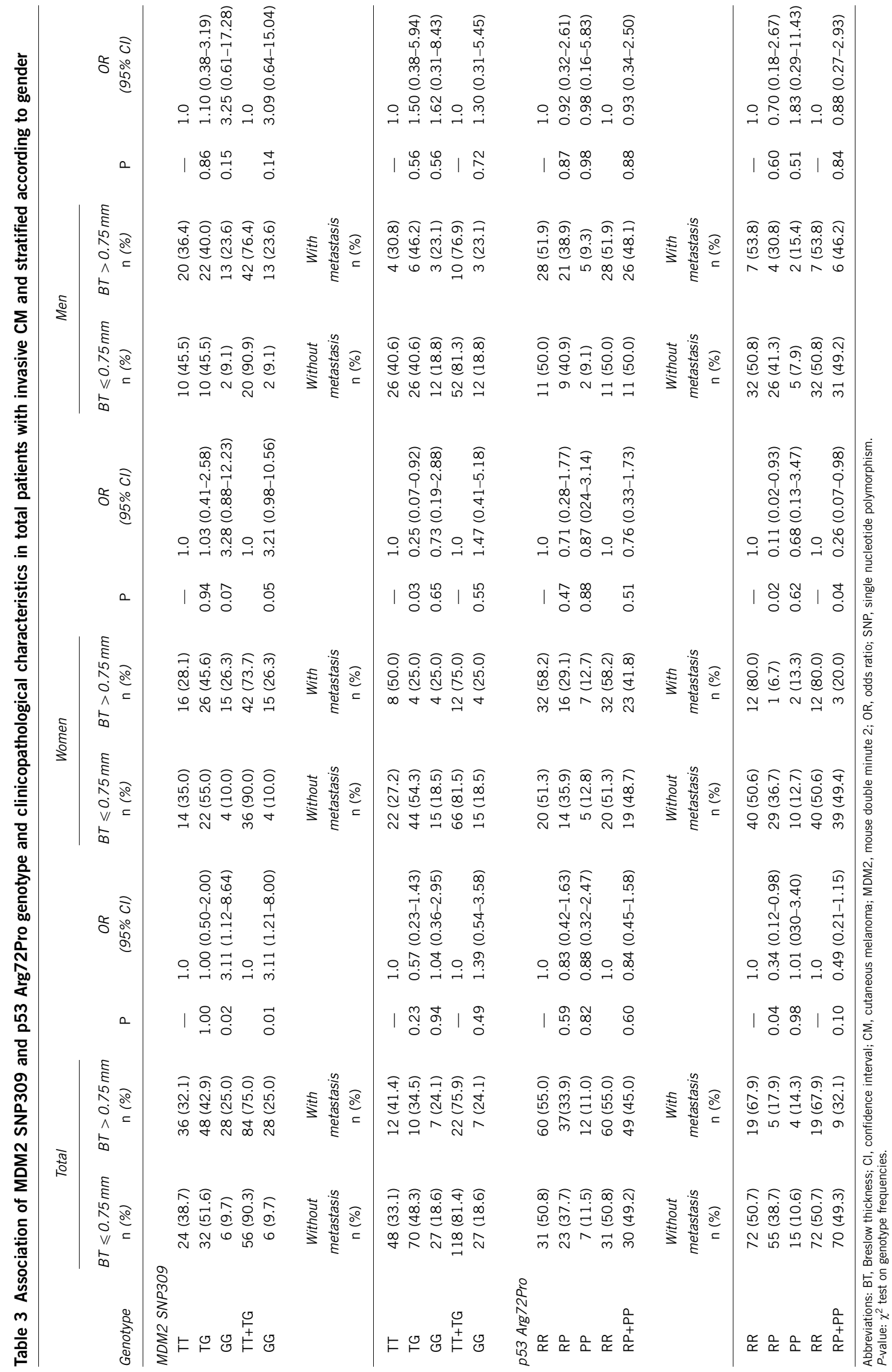


clarified in follow-up replication studies having information regarding the menopausal status at the time of melanoma, missing in this and the other two previously published studies. ${ }^{10,11}$

Breslow thickness is the most powerful histopathological predictor of melanoma progression currently known. ${ }^{25,30,31}$ Therefore, investigation of MDM2 SNP309 genotypes in terms of tumor Breslow thickness was of particular interest. SNP309 genotypes were compared among patients stratified according to the clinically significant cutoff point of $0.75 \mathrm{~mm} .^{25}$ The SNP309 GG genotype appeared to be associated with tumors thicker $(>0.75 \mathrm{~mm})$ when compared with TT and TG, supporting the evidence that the GG genotype may confer risk for increasing of melanoma tumor while both TT and TG genotype may have a protective effect. This genetic association was not found to be gender specific.

The median tumor Breslow thickness of GG carriers was found significantly increased. Firoz et al. evaluated the association between median tumor thickness and SNP309 genotype in 89 women and 127 men with CM separately, and no significant associations were found. ${ }^{10}$ However, the median of GG carriers was higher than that of TT carriers for both women and men. They did not mention whether analyses based on the entire population (women and men pooled), genetic models of association and patients stratified according to a clinically significant cutoff were performed.

From this preliminary study, the SNP309 GG genotype in the Italian melanoma population therefore seems to correlate with a poor prognostic indicator, although a larger cohort would be required to verify these findings. However, this finding seems to be in line with the well-documented functional relevance of this SNP. A model was proposed that the G-allele in the promoter of $M D M 2$ increases the DNA-binding affinity of the transcriptional activator, Sp1, which results in high levels of MDM2 mRNA and protein in human cells and the subsequent attenuation of the p53 pathway that favors the tumor formation. ${ }^{4}$ Furthermore, patients with B-cell chronic lymphocytic leukemia and renal cell carcinoma carrying the GG genotype were reported to have higher levels of MDM2 protein in tumor cells and to show worse prognosis and low survival. ${ }^{14,32}$ We thus hypothesize that the CM patients carrying the GG genotype may have higher levels of MDM2 and so a subsequent attenuation of the p53 pathway could favourite a worst prognosis. This hypothesis is supported by the fact that $\mathrm{CM}$ belongs to a group of tumors for which p53 mutations are rare $(0-10 \%)$, indicating that other events are contributing to p53 inactivation. ${ }^{33}$ The genetic model of MDM2 SNP309 proposed by Bond et al. might be one of these events. ${ }^{4}$ Further efforts are clearly required to elucidate the potential role of SNP309 in CM.

The results of this paper suggest that, in a model of recessive inheritance, SNP309 G allele has no effect on the risk of CM, but it might influence the prognosis. These are in line with a recently published meta-analysis on MDM2 SNP309 and cancer risk. ${ }^{9}$ It supports the evidence that this polymorphism, based on a recessive model for the $\mathrm{G}$ allele, has little or no effect on the risk of common cancers, but it might modify the time of tumor onset and prognosis. ${ }^{9}$

Studies in other cancers have reported a combined effect of both MDM2 and p53 polymorphisms on cancer risk and survival. ${ }^{12-14}$ In our study, we found no association with risk of $\mathrm{CM}$ when the genotypes of the two SNPs were combined. The effect of p53 Arg72Pro on melanoma remains controversial, with some studies reporting associations between melanoma and the PP genotype ${ }^{17,19}$ and others reporting associations between melanoma and the RR genotype. ${ }^{18,20}$ Moreover, here we report that the distribution of p53 Arg72Pro genotypes do not vary by age, gender or Breslow thickness.
A significant increase of the TG and RP genotype frequencies were observed in women without metastasis. We have no immediate biological explanation for this sex-specific association. It may have been due to chance in the subgroup analysis, as the number of patients with metastasis was low (total 33). Nonetheless, this finding warrant further studies in different and larger populations.

In conclusion, within this Italian population, the MDM2 309 polymorphism was found to associate with thick tumors (Breslow thickness $>0.75 \mathrm{~mm}$ ) but had no impact on the age of diagnosis and the presence of metastasis for CM. However, as the Breslow thickness is widely considered as the most important single prognostic indicator in melanoma, this finding might have, in future, implication in improving the management of melanoma with performing a more accurate diagnosis and more precise programs of prevention of disease. Furthermore, this study confirms that the SNP309 is not associated with the risk of CM as already demonstrated in a Caucasian female population.

\section{ABBREVIATIONS}

$\mathrm{CM}$, cutaneous melanoma; $\mathrm{CI}$, confidence interval; OR, odds ratio; SNP, single nucleotide polymorphism.

\section{CONFLICT OF INTEREST}

The authors declare no conflict of interest.

\section{ACKNOWLEDGEMENTS}

This study was supported by AIRC, MURST-PRIN, OPEN foundation, Ministero Sanità and Telethon. The salary of Dr Mario Capasso was supported by AIRC-FIRC. Furthermore, this work is partially supported by grants from MIUR (P35/126/IND) and Regione Campania (DGRC 1901/2009). We thank all the patients and healthy blood donors for providing blood samples.

1 Momand, J., Zambetti, G. P., Olson, D. C., George, D. \& Levine, A. J. The mdm-2 oncogene product forms a complex with the p53 protein and inhibits p53-mediated transactivation. Cell 69, 1237-1245 (1992).

2 Michael, D. \& Oren, M. The p53-Mdm2 module and the ubiquitin system. Semin. Cancer Biol. 13, 49-58 (2003).

3 Zhang, Z. \& Zhang, R. p53-independent activities of MDM2 and their relevance to cancer therapy. Curr. Cancer Drug Targets 5, 9-20 (2005).

4 Bond, G. L., Hu, W., Bond, E. E., Robins, H., Lutzker, S. G., Arva, N. C. et al. A single nucleotide polymorphism in the MDM2 promoter attenuates the p53 tumor suppressor pathway and accelerates tumor formation in humans. Cell 119, 591-602 (2004).

5 Harris, S. L., Gil, G., Robins, H., Hu, W. Hirshfield, K., Bond, E. et al. Detection of functional single-nucleotide polymorphisms that affect apoptosis. Proc. Natl Acad. Sci. USA 102, 16297-16302 (2005).

6 Millikan, R. C., Heard, K. Winkel, S., Hill, E. J., Heard, K. Massa, B. et al. No association between the MDM2 -309 T/G promoter polymorphism and breast cancer in African-Americans or Whites. Cancer Epidemiol. Biomarkers Prev. 15, 175-177 (2006).

7 Park, S. H., Choi, J. E., Kim, E. J., Jang, J. S., Han, H. S., Lee, W. K. et al. MDM2 309T $>$ G polymorphism and risk of lung cancer in a Korean population. Lung Cancer 54, 19-24 (2006).

8 Toyama, T., Zhang, Z., Nishio, M., Hamaguchi, M., Kondo, N., Iwase, H. et al. Association of TP53 codon 72 polymorphism and the outcome of adjuvant therapy in breast cancer patients. Breast Cancer Res. 9, R34 (2007).

9 Wilkening, S., Bermejo, J. L. \& Hemminki, K. MDM2 SNP309 and cancer risk: a combined analysis. Carcinogenesis 28, 2262-2267 (2007)

10 Firoz, E. F., Warycha, M., Zakrzewski, J., ollens, D., Wang, G., Shapiro, R. et al. Association of MDM2 SNP309, age of onset, and gender in cutaneous melanoma. Clin. Cancer Res. 15, 2573-2580 (2009).

11 Nan, H., Qureshi, A. A., Hunter, D. J. \& Han, J. A functional SNP in the MDM2 promoter, pigmentary phenotypes, and risk of skin cancer. Cancer Causes Control 20, 171-179 (2009)

12 Hong, Y., Miao, X., Zhang, X., Ding, F., Luo, A., Guo, Y. et al. The role of P53 and MDM2 polymorphisms in the risk of esophageal squamous cell carcinoma. Cancer Res. 65, 9582-9587 (2005).

13 Zhang, X., Miao, X., Guo, Y., Tan, W., Zhou, Y., Sun, T. et al. Genetic polymorphisms in cell cycle regulatory genes MDM2 and TP53 are associated with susceptibility to lung cancer. Hum. Mutat. 27, 110-117 (2006). 
14 Hirata, H., Hinoda, Y., Kikuno, N., Kawamoto, K., Suehiro, Y., Tanaka, Y. et al. MDM2 SNP309 polymorphism as risk factor for susceptibility and poor prognosis in renal cell carcinoma. Clin. Cancer Res. 13, 4123-4129 (2007).

15 Talseth, B. A., Meldrum, C., Suchy, J., Kurzawski, G., Lubinski, J. \& Scott, R. J. MDM2 SNP309 T $>\mathrm{G}$ alone or in combination with the TP53 R72P polymorphism does not appear to influence disease expression and age of diagnosis of colorectal cancer in HNPCC patients. Int. J. Cancer 120, 563-565 (2007).

16 Schmidt, M. K., Reincke, S., Broeks, A., Braaf, L. M., Hogervorst, F. B., Tollenaar, R. A. et al. Do MDM2 SNP309 and TP53 R72P interact in breast cancer susceptibility? A large pooled series from the breast cancer association consortium. Cancer Res. 67, 9584-9590 (2007).

17 Stefanaki, I., Stratigos, A. J., Dimisianos, G., Nikolaou, V., Papadopoulos, O., Polydorou, D. et al. p53 codon 72 Pro homozygosity increases the risk of cutaneous melanoma in individuals with dark skin complexion and among noncarriers of melanocortin 1 receptor red hair variants. Br. J. Dermatol. 156, 357-362 (2007).

$18 \mathrm{Li}$, C., Chen, K., Liu, Z., Wang, L. E., Gershenwald, J. E., Lee, J. E. et al. Polymorphisms of TP53 Arg72Pro, but not p73 G4C14 >A4TA4 and p21 Ser31Arg, contribute to risk of cutaneous melanoma. J. Invest. Dermatol. 128, 1585-1588 (2008).

19 Gwosdz, C., Scheckenbach, K., Lieven, O., Reifenberger, J., Knopf, A., Bier, H. et al. Comprehensive analysis of the p53 status in mucosal and cutaneous melanomas. Int. J. Cancer 118, 577-582 (2006).

20 Shen, H., Liu, Z., Strom, S. S., Spitz, M. R., Lee, J. E., Gershenwald, J. E. et al. p53 codon 72 Arg homozygotes are associated with an increased risk of cutaneous melanoma. J. Invest. Dermatol. 121, 1510-1514 (2003).

21 Chung, E. S., Sabel, M. S. \& Sondak, V. K. Current state of treatment for primary cutaneous melanoma. Clin. Exp. Med. 4, 65-77 (2004).

22 Balato, N., Gaudiello, F., Balato, A. \& Monfrecola, G. Sun habits in the children of Southern Italy. J. Am. Acad. Dermatol. 57, 883-887 (2007).

23 Carli, P., Naldi, L., Lovati, S. \& La Vecchia, C. The density of melanocytic nevi correlates with constitutional variables and history of sunburns: a prevalence study among Italian schoolchildren. Int. J. Cancer 101, 375-379 (2002).
24 Paulin, F. E., O'Neill, M., McGregor, G., Cassidy, A., Ashfield, A., Ali, C. W. et al. MDM2 SNP309 is associated with high grade node positive breast tumours and is in linkage disequilibrium with a novel MDM2 intron 1 polymorphism. BMC Cancer 8, 281 (2008).

25 Breslow, A. Thickness, cross-sectional areas and depth of invasion in the prognosis of cutaneous melanoma. Ann. Surg. 172, 902-908 (1970).

26 Capasso, M., Ayala, F., Russo, R., Avvisati, R. A., Asci, R., Iolascon, A. A predicted functional single-nucleotide polymorphism of bone morphogenetic protein-4 gene affects mRNA expression and shows a significant association with cutaneous melanoma in Southern Italian population. J. Cancer Res. Clin. Oncol. 135, 1799-1807 (2009).

27 Mocellin, S., Verdi, D. \& Nitti, D. DNA repair gene polymorphisms and risk of cutaneous melanoma: a systematic review and meta-analysis. Carcinogenesis 30, 1735-1743 (2009).

28 Bond, G. L., Hirshfield, K. M., Kirchhoff, T., Alexe, G., Bond, E. E., Robins, H. et al. MDM2 SNP309 accelerates tumor formation in a gender-specific and hormonedependent manner. Cancer Res. 66, 5104-5110 (2006).

29 Bond, G. L., Menin, C., Bertorelle, R., Alhopuro, P., Aaltonen, L. A. \& Levine, A. J. MDM2 SNP309 accelerates colorectal tumour formation in women. J. Med. Genet. 43, 950-952 (2006).

30 Balch, C. M., Soong, S. J., Gershenwald, J. E., Thompson, J. F., Reintgen, D. S., Cascinelli, N. et al. Prognostic factors analysis of 17,600 melanoma patients: validation of the American Joint Committee on Cancer melanoma staging system. J. Clin. Oncol. 19, 3622-3634 (2001).

31 Fortes, C. Mastroeni, S., Sera, F., Concolino, F., Abeni, D. Melchi, F. et al Survival and prognostic variables of cutaneous melanoma observed between 1995 and 2000 at Istituto Dermopatico Dell'Immacolata (IDI-IRCCS), Rome, Italy. Eur. J. Cancer Prev. 15, 171-177 (2006)

32 Gryshchenko, I., Hofbauer, S., Stoecher, M., Daniel, P. T., Steurer, M., Gaiger, A. et al. MDM2 SNP309 is associated with poor outcome in B-cell chronic lymphocytic leukemia. J. Clin. Oncol. 26, 2252-2257 (2008).

33 Box, N. F. \& Terzian, T. The role of p53 in pigmentation, tanning and melanoma. Pigment Cell Melanoma Res. 21, 525-533 (2008). 